\title{
Monitorización de parámetros de calidad de redes de transmisión mediante alta resolución espectral en dominio óptico.
}

\author{
P. Sevillano, J. Subías \\ Grupo de Tecnologías Fotónicas (GTF) \\ Instituto de Investigación en Ingeniería de Aragón (I3A) \\ Universidad de Zaragoza, Mariano Esquillor s/n, 50018, Zaragoza, Spain. \\ Tel. +34-976762707, Fax +34-976762043, e-mail: pascual.sevillano@unizar.es
}

\begin{abstract}
La creciente demanda de mayor ancho de banda en los sistemas de comunicación globales exige una arquitectura cada vez mas compleja donde toman fuerza aquellas redes en las cuales se prescinde de puntos de regeneración intermedios. Los sistemas con multiplexación densa en longitud de onda (DWDM) de camino reconfigurable exigen una revisión de los estándares de medida de parámetros de calidad como la dispersión del modo de polarización (PMD) o la relación señal a ruido óptica (OSNR). Se describen en este trabajo las posibilidades que ofrece el análisis de espectros mediante difusión Brillouin estimulada para la monitorización de PMD y OSNR frente a métodos convencionales. El uso de la difusión Brillouin estimulada como filtro en un analizador de espectros ópticos permite obtener valores de densidad de potencia en intervalos espectrales suficientemente estrechos como para no verse alterados por la proximidad entre canales. El análisis espectral de alta resolución introduce mejoras significativas en los métodos de medida de OSNR basados en la supresión de señal por polarización, al hacerlos compatibles con variaciones del estado de polarización a lo largo del ancho de banda de la señal. Además, la coherencia intrínseca del efecto de dispersión Brillouin permite monitorizar diferencias en estado de polarización entre componentes espectrales de la señal, y caracterizar de esta manera en dominio óptico la degradación por PMD presente en cada uno de los canales de una señal problema sin necesidad de recurrir a métodos en detección como el diagrama de ojo o la tasa de error de bit.
\end{abstract}

